\title{
GLOBALIZACIÓN, GRANDES PROYECTOS Y PRIVATIZACIÓN DE LA GESTIÓN URBANA
}

Mario Lungo Díaz ${ }^{1}$

\section{Homenaje a Mario Lungo}

Revista Urbano, ha querido hacer un homenaje póstumo al autor de este significativo texto, el Arquitecto salvadoreño don MARIO LUNGO, quien recientemente dejara de existir en su patria natal, luego de una penosa enfermedad.

El profesor Lungo, era un profesional conocido de nuestra Universidad y un gran amigo de Chile y recientemente, nos había mandado este texto pocas semanas antes que enfermara gravemente.

$\mathrm{Su}$ aporte profesional y académico en su país y en Centroamérica, ha sido reconocido ampliamente, no sólo por sus ideas lúcidas y su trabajo incansable, sino por su calidad humana y su fuerte defensa de los valores cívicos y de los derechos de los más pobres. Mario fue un urbanista de la ciudad Democrática y Solidaria, un legado pendiente en nuestra sur-ámerica, aún carente de justicia y equidad. Confiamos en que ese llamado sea fecundo y escuchado por las nuevas generaciones que deberán completar su trabajo.

Desde Concepción, al sur de Chile, le enviamos a Patricia, su esposa, a su familia, a sus amigos y a sus numerosos alumnos, nuestro pesar por esta partida irreparable. Lo seguiremos recordando en sus textos y en la ideas que seguirán discutiéndose en la búsqueda de las ciudades que él soñó.

Juan Pablo Gramsch Labra

Consejo Editor Revista Urbano

Revista URBANO se siente muy honrada en publicar este trabajo póstumo del destacado Arquitecto y Profesor don Mario Lungo, quien tuviera la gentileza de enviarnos este trabajo en sus últimos meses de vida.

Estas páginas, intentarán ser fieles a los postulados y principios de este gran Maestro y cultor de nuestra disciplina urbana. Vaya para él nuestro sentido homenaje y agradecimiento por distinguirnos e iluminarnos con sus verdades e ideales.

Alfredo Palacios Barra

Editor Revista URBANO

\section{INTRODUCCIÓN}

El presente artículo tiene por objetivo contribuir a discutir si los grandes proyectos urbanos, o grandes intervenciones urbanas, que se han convertido en un instrumento clave de la planificación de las ciudades en Europa en las últimas dos décadas, y comienza a ser incorporado en la planificación de las ciudades latinoamericanas recientemente, constituye una vía para impulsar la privatización de le gestión urbana en nuestros países y adecuarla al enfoque neoliberal de las políticas públicas hoy predominante.

Este reflexión nace de la preocupación por la crisis de la planificación urbana y de los intentos de privatización de la gestión urbana en general que se observa desde hace algunos años, pero a la vez del análisis de la ejecución de grandes proyectos urbanos en América Latina que muestra tendencias y efectos contradictorios, lo que exige una posición cuidadosa al respecto. (Nota: Esta reflexión se apoya en las discusiones realizadas en el marco de los cursos latinoamericanos sobre Grandes Proyectos Urbanos, organizados por el Lincoln Institute of Land Policy, de Cambridge, Massachusetts en los años 2002, 2003 y 2004).

\section{Globalización y gestión urbana}

Desde finales de la década de los 80 se planteó que se estaba operando una transformación en la administración de las ciudades, pasándose de una modalidad de gerenciamiento a otra donde predominaba una visión empresarial (Harvey, 1996). Este autor parte de que a partir de los años 70 parece hacerse establecido, en los países del capitalismo avanzado, un consenso sobre los beneficios positivos se obtendrían si las ciudades asumían un comportamiento empresarial en relación al desarrollo económico, que esta visión tenía que ver con la declinación del poder de los estados nacionales para controlar los flujos monetarios internacionales y que, en el fondo, expresaba la dinámica general de la transformación del modelo de acumulación fordista-keynesiano hacia un régimen de acumulación flexible.

Señala que una de los instrumentos centrales de la gestión urbana empresarial son las asociaciones público-privadas, que tienen una base esencialmente especulativa, mientras su foco de atención se orienta más a la economía local que al territorio. Para la gestión urbana empresarial existen cuatro condiciones básicas: la exploración de las ventajas específicas para la producción de bienes y servicios; aumentar la competitividad a partir de la división espa- 
cial del consumo; el control de las operaciones financieras de mayor importancia; y la competencia por el excedente del gobierno central.

Lo anterior conduce a una situación contradictoria: existen límites a la gestión urbana empresarial debido a la generación de una intensa competencia entre las ciudades que tiende acentuar el desarrollo territorial desigual, al interior de cada ciudad y entre ellas. Sin embargo Harvey sostiene que debe analizarse su potencial para transformarse en un corporativismo urbano progresista capaz de construir alianzas para mitigar e incluso desafiar a la dinámica hegemónica de la acumulación capitalista.

En un trabajo escrito a principios de los años 90 (Lungo, 1992), planteábamos que en estrecha asociación con el modelo de desarrollo basado en la sustitución de importaciones y la ampliación del aparato estatal y sus políticas que se impulso a mediados del siglo XX, la planificación urbana tenía dos rasgos esenciales: su carácter altamente regulador y su cobertura global, bajo las que subyacía un sentido de redistribución de la riqueza. Esto marca decisivamente el enfoque de sus objetivos y la búsqueda de un "modelo de ciudad ideal" hacia el cual se pretendía llegar, y lo que es más importante, los instrumentos y la formación del personal para alcanzar la meta buscada. Casi totalmente apoyada en la intervención de la tecnocracia del gobierno central, el papel de los gobiernos locales es prácticamente ignorado y la participación popular, cuando se plantea, es una participación tutelada.

Bastante tiempo antes que la crisis de finales de los años 70 mostrara el agotamiento del modelo de sustitución de importaciones, esta concepción de planificación del desarrollo urbano había perdido casi toda su vigencia abriéndose un período de incertidumbre en torno a cómo manejar los problemas generados por el continuado crecimiento de las ciudades. Nos vamos a encontrar, sin embargo, un claro debate al respecto. La extrema complejidad de los procesos y problemas urbanos impide pensar en un total abandono del papel del Estado, por lo que, globalmente, no se da una confrontación entre planificación urbana estatal y mercado, sino que podemos observar una cambiante relación entre ellos, relación que sigue en cada país un particular movimiento.

En términos generales podemos observar un giro a mediados de los años 70, cuando se pasa de la planificación urbana, centrada especial aunque no exclusivamente, en las ciudades y la totalidad de sus procesos, a pensar en planificación en términos de componentes de la estructura física y socio-económica urbana directa y casi exclusivamente relacionadas con la población de menores ingresos. Este giro, podríamos plantear, constituye un paso intermedio hacia la configuración de un modelo de gestión urbana en que el papel del mercado y la desregulación son las características centrales.

Encontramos la expresión de este cambio en un importante documento de la Naciones Unidas, publicado la siguiente década y que sistematiza la experiencia de 10 años en el impulso de esta nueva visión de abordar los problemas urbanos. (ONU-HÁBITAT, 1986). Constatando las limitaciones de la planificación convencional frente a los cambios operados en el ámbito urbano que mostraban numerosos estudios, en los que problemas como la migración rural-urbana, la pobreza y la informalización de la economía dejan de verse sólo en sus aspectos negativos, se comienzan

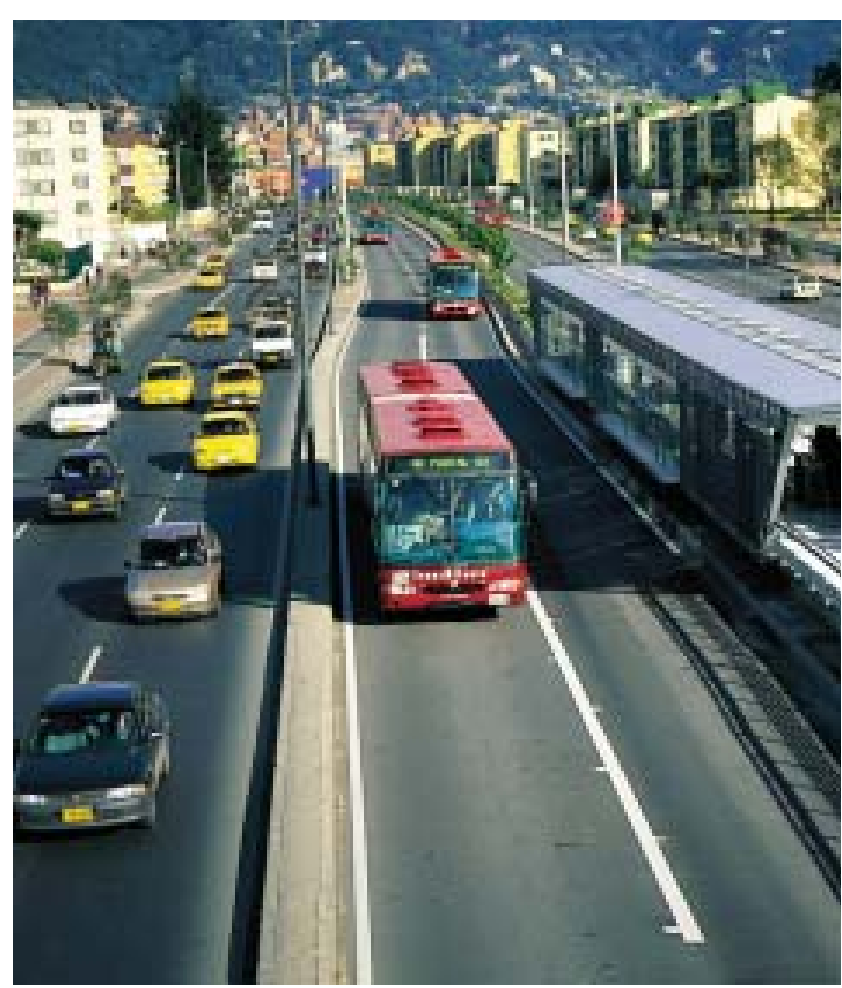

M oderno servicio de transporte urbano. Plan Transmilenio, Bogotá, Colombia.

a visualizar los asentamientos precarios urbanos también por la contribución económica de sus habitantes en tanto fuerza de trabajo, consumidores y productores de infraestructura física y vivienda, además del alto nivel de su integración social, cultural y política.

La planificación ya no de la ciudad en su totalidad sino de estos asentamientos precarios pasa a convertirse en el eje de una nueva forma de ver la planificación urbana en que el resto de procesos y problemas deja de ser sujeto privilegiado de la intervención estatal aunque ella no desaparezca. Inmersa en un contexto de crisis fiscal, esta nueva visión de la planificación urbana orientará sus acciones a cuestiones antes no totalmente atendidas como los programas de mantenimiento y rehabilitación de infraestructura física urbana y a repensar el financiamiento, dentro del cual se comienza a cuestionar cada día más la inversión pública, promoviendo la recolección de fondos por parte de los gobiernos locales y nuevas formas de movilización del ahorro privado.

Las intervenciones se sectorializan mientras van adecuándose a las condiciones económicas y políticas imperantes; así por ejemplo, se plantean nuevas intervenciones en torno a la tierra urbana, unas a través de reformas jurídicas y otras a través del reordenamiento en su utilización: redefinición del uso de la tierra urbana, modernización del catastro y del registro, cambios en la legislación, modificación en los mecanismos de intervención pública, utilización de la tierra urbana pública, etc. Esta última (en el sentido de su venta), y la modernización catastral serán las acciones privilegiadas.

Sin embargo, frente al énfasis excesivo de los trámites burocráticos, las propuestas hacia la formulación de una nueva manera de enfrentarse a los problemas urbanos priorizarán la concesión a los grupos de base la mayor autonomía posible para resolverlos, en 
un discurso ligado a la democratización, al reforzamiento de los gobiernos locales y al papel de la sociedad civil. Esta presenta dos grandes limitaciones: por un lado, la ejecución de innumerables acciones aisladas sin un plan de desarrollo global que las oriente; por otro lado, la atomización de las reivindicaciones de los sectores populares urbanos mientras el resto de los procesos urbanos son cada día menos regulados. La acentuación de contradicciones urbanas, viejas y nuevas, se dejan así en manos del mercado cada día más, modificándose las condiciones en la relación público-privado en torno a aspectos como los servicios urbanos.

La participación del Estado cambia así radicalmente. Se trata ahora de una función de apoyo y no de regulación o inversión. Se trata de organizar la información y la comunicación, de dar entrenamiento, de reforzar las organizaciones de base, de apoyar el papel de las ONG's, etc., dentro de un esquema de flexibilidad en la planificación y la implementación de programas. La planificación, ya no de la ciudad, sino de los asentamientos humanos es parte así de las nuevas concepciones del crecimiento económico y del desarrollo sostenible, dentro de las cuales se estaría configurando una nueva forma de gestión urbana que se asocia al modelo económico neoliberal predominante.

El Banco Mundial insistió mucho hasta finales de los 90 en el carácter temporal de los efectos negativos de los programas de ajuste estructural sobre los sectores pobres, por lo que sus acciones en las áreas urbanas han tenido un enfoque focalizante de otros programas sociales. Hoy reconoce esta institución la persistencia de estos efectos negativos pero continua impulsando la opción de desregulación y privatización de los programas y servicios sociales urbanos, siendo esta tendencia uno de los pilares más importante en la configuración de una nueva manera de enfrentarse a los problemas del desarrollo de nuestras ciudades (Banco Mundial, 1999 y 2000).

Subyace en esta visión el supuesto, no totalmente erróneo, de que las grandes ciudades no son tanto un problema sino una fuente de producción de riqueza y que lo que se debe hacer es incrementar la "productividad urbana" (noción por otra parte llena de enorme ambigüedad), venciendo los obstáculos siguientes: las deficiencias de la infraestructura urbana; la existencia de regulaciones inapropiadas; la debilidad de las finanzas municipales; y la debilidad de los sistemas financieros nacionales para

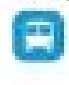

Veseaclaives de buses movilizar recursos para el desarrollo urbano.

Una idea quisiéramos destacar en torno a esta nueva forma de gestión urbana: dentro de ella una cuestión clave sigue siendo el suelo urbano; por ello, la visión neoliberal de la gestión urbana tiene, a través de la estructuración del mercado de tierra urbana, una de sus principales vías de acción.

Plantear la construcción de una propuesta alternativa de desarrollo de las ciudades que se oponga a esta concepción neoliberal de la gestión urbana exige superar el dilema siguiente: ¿¿Dentro de qué modelo de desarrollo económico social y dentro de qué visión de la ciudad por construir se insertaría esta propuesta alternativa de gestión urbana? Creemos que no es posible, que es además erróneo, pretender tener una visión acabada de la sociedad y de la ciudad que se pretende construir. Sin negar la necesidad y la

\section{Sistema Integrado de Transporte
Urbano para el Gran Concepción}

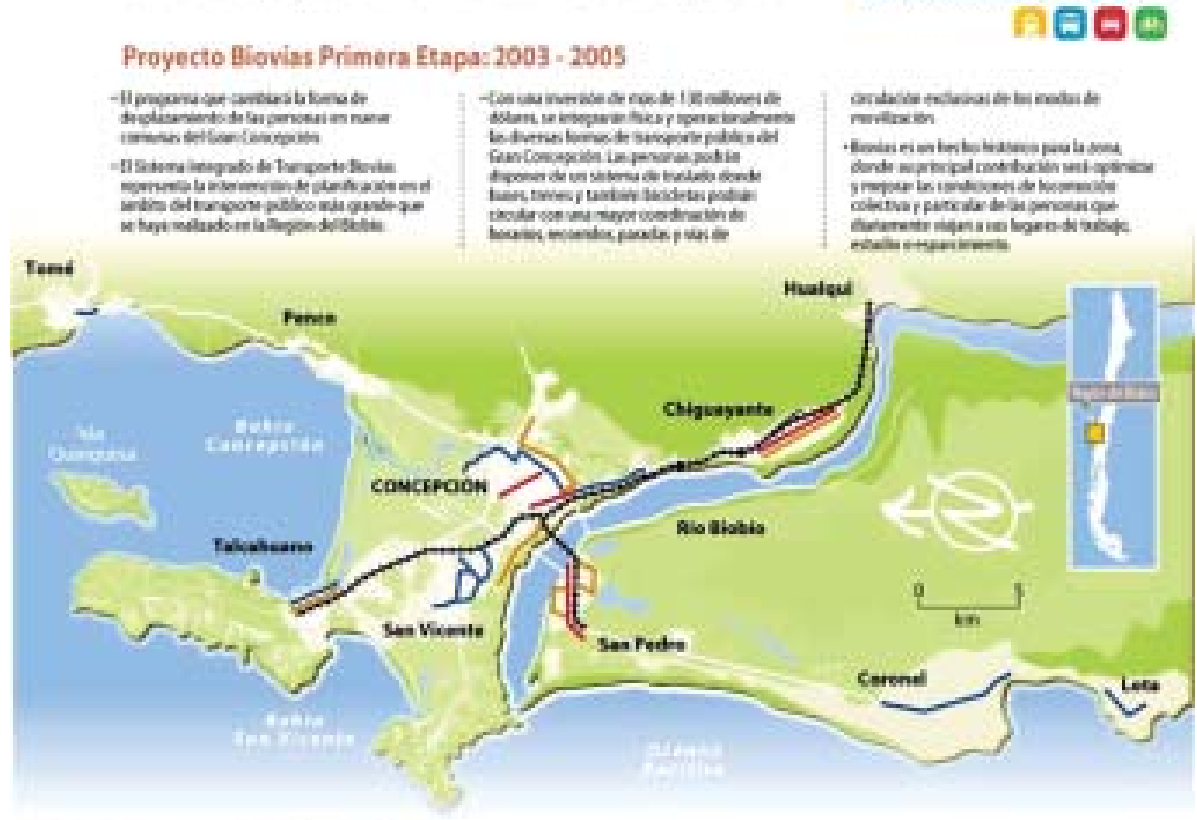

Priscipalirs componentes del Fias Sionios.

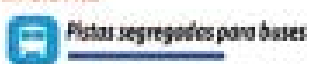

Treses decerravie

Find decicloniti

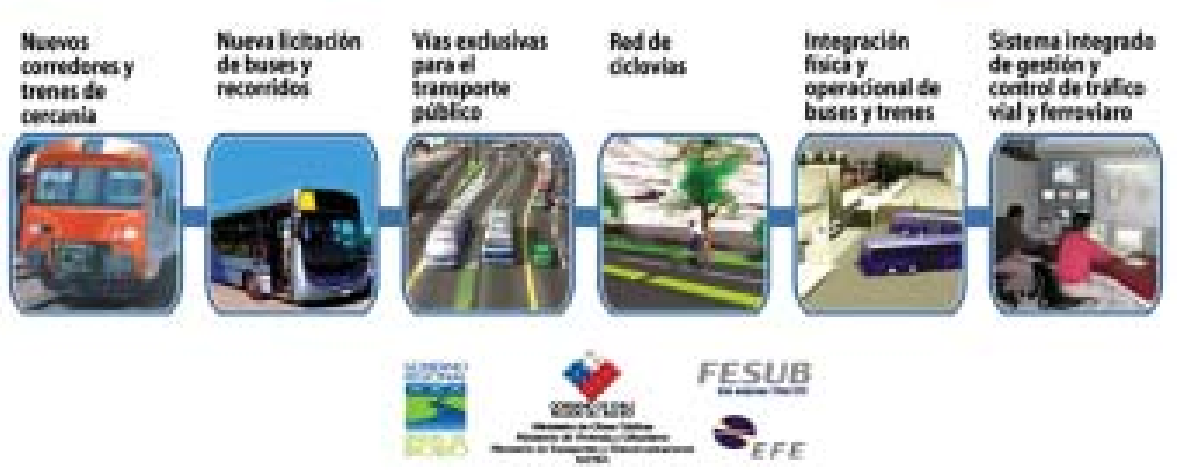

Proyecto Biovías, sistema integrado de transporte urbano, Concepción. 
validez de las utopías, sostenemos que la construcción de una nueva sociedad de una nueva ciudad, es un proceso permanente y contradictorio de construcción ininterrumpida que asume formas especificas en cada caso concreto.

¿Cuál sería la estrategia a seguir para ir construyendo una forma alternativa de gestión de nuestra ciudades que se oponga a la desregulación y privatización indiscriminadas, y también a la atomización de las acciones puntuales? Pensamos que ella debería incorporar acciones de cuatro tipos:

- Primero, es necesario destacar y conocer cuáles son los procesos claves de la estructuración urbana en la fase actual. Aquí aparece la necesidad de dar un nuevo impulso a la investigación urbana, en el sentido de reformulación y profundización teórica y una mayor articulación con la información empírica.

- Segundo, conociendo las características de la nueva estructura social urbana, se debe buscar el establecimiento de alianzas y consensos con los distintos sectores en torno a la ciudad que se busca construir y la forma de irlo logrando. Debe superarse la visión errada que piensa sólo en los pobres, obviando el hecho de la complejidad social de la ciudad y que una legítima opción de desarrollo urbano que favorezca a los sectores populares y busque la construcción de una ciudad más justa no puede olvidar la multiplicidad de intereses que se expresan en ella. - Tercero, se deben construir espacios de participación y decisión políticos permanentes a través de los cuales los sectores populares puedan articular sus múltiples reivindicaciones, evitar su dispersión y superposición, y potenciar su poder de decisión.

- Cuarto, estos espacios de poder construidos deben ser la base para ir impulsando las medidas irreversibles que permitan ir construyendo la ciudad democrática que se busca.

Porque si es cierto que la forma de gestión urbana prevaleciente durante las décadas anteriores estaba prácticamente agotada a finales de los años 80 y se vislumbran "horizontes críticos" en torno a numerosos problema urbanos (Prefeitura de Sao Paulo, 1991), la construcción de una propuesta alternativa a una concepción neoliberal de la gestión urbana pasa por superar el esquematismo de posiciones "de principio" en torno al problema de la privatización de los servicios urbanos, por ejemplo. Sólo una conducción pública del desarrollo de la ciudad participativa y articulada estratégicamente alrededor de los procesos urbanos fundamentales, y que asuma flexiblemente la relación entre o público y lo privado, podrá oponerse a la opción de gestión urbana neoliberal.

\section{Los grandes proyectos urbanos y su papel en la gestión urbana}

En esta lucha aparece una modalidad de construcción de la ciudad que es importante discutir: la planificación urbana apoyada en la ejecución de grandes proyectos urbanos. Al hablar de éstos estamos ante una noción ambigua y contradictoria, y por esta razón elusiva (Lungo, 2002 y 2004). No se pretende aquí elaborar un concepto definitivo, únicamente abrir la discusión acerca de lo que se entiende, desde diferentes visiones, por estas grandes intervenciones en las ciudades.
Estas comprenden no sólo las grandes obras emblemáticas, sino también grandes operaciones urbanas que asumen, en muchos casos, la forma de programas de intervención a través de un conjunto acciones urbanas de nivel intermedio que por su integración tienen un profundo impacto en el desarrollo de una ciudad. La importancia del estudio de los grandes proyectos u operaciones urbanas en el momento actual deriva de la profunda transformación que está generando el proceso de globalización, redefiniendo las funciones de las ciudades y colocando la cuestión de la competitividad de las mismas, con todo lo problemático y discutible que significa la aplicación de este concepto económico para el desarrollo urbano, en un primer plano en la planificación del desarrollo de las ciudades.

Aunque a lo largo de las últimas décadas en las ciudades latinoamericanas encontramos numerosos proyectos u operaciones de gran envergadura, no se ha elaborado, ni en los estudios urbanos ni en planificación urbana tradicional, una conceptualización específica sobre éstos. Es hasta en los últimos años, con la introducción del enfoque de la planificación estratégica, cuando éstos pasan a ocupar una posición privilegiada como acciones claves para impulsar el desarrollo de las ciudades, que se comienza a elaborarse su conceptualización. Existe, no obstante, un obstáculo a considerar: la enorme diversidad de este tipo de proyectos, lo que plantea un importante desafío analítico.

Estos proyectos pueden ser la recuperación de centros históricos; la construcción de sistemas de transporte público masivos, la reutilización de antiguas zonas industriales, militares, ferroviarias, portuarias, aeroportuarias, etc.; la rehabilitación de grandes áreas de vivienda degradada (Prefeitura de Rio de Janeiro, 1999); la construcción de nuevas zonas turísticas o recreativas; etc. A esta diversidad se agregan los problemas de la definición de la escala y la multiplicidad de actores presentes que caracterizan a este tipo de proyectos urbanos. Aquí encontramos una diferencia del contenido de esta temática en América Latina con relación a los Estados Unidos y Europa. En la primera se incluyen intervenciones urbanas de muy distinto tipo y con diferentes objetivos como los mencionados antes, mientras que en Los Estados Unidos prevalecen las grandes construcciones de infraestructura urbana y en Europa la discusión se orienta los grandes proyectos urbanos de corte emblemático.

En esta diversidad encontramos factores determinantes y lógicas de actuación muy diferentes que obedecen a la compleja heterogeneidad de los intereses presentes, dificultando la conceptualización de los llamados genéricamente grandes proyectos urbanos.

La noción de proyecto urbano surge en Europa en los años 70 , asociada a la contradicciones existentes entre los proyectos arquitectónicos de gran dimensión y los planes urbanos. Posteriormente el proyecto urbano pasa a ser entendido como una actuación pública sobre un segmento de la ciudad pero articulada a una visión global de la misma y a los problemas económicos y sociales. Con este cambio la noción de proyecto urbano compite con la de plan urbano al ser más atractiva para los inversionistas privados al precisar las propuestas espaciales e incluir, además de aspectos económicos claves, objetivos sociales, culturales y ambientales, y exigir el acuerdo de múltiples actores, entre ellos los privados. A partir 
de este cambio en la noción de proyecto urbano esté se impone en el urbanismo europeo desde los años 80 , generando diferentes propuestas analíticas (Ingallina, 2001).

Consideramos que la clave para el análisis de este tipo de intervenciones está en su complejidad económica y social y no sólo en la escala espacial, aunque ésta sea una condición necesaria, lo que introduce mayores problemas para construir una tipología de grandes proyectos urbanos o indicadores específicos para el análisis de éstos.

El gran proyecto urbano es entonces una noción polisémica, pero se puede entender como el hecho de articular una estrategia fuerte alrededor de apuestas urbanas y socioeconómicas de gran dimensión y larga duración que inciden en el desarrollo de una ciudad construido socialmente, cuando ésta exista. No debe, sin embargo, limitarse a una acción urbana específica como un proyecto arquitectónico de gran dimensión, ni confundirse con el proyecto de ciudad.

Los grandes proyectos urbanos constituyen uno de los rasgos claves del urbanismo europeo de finales del siglo XX, aunque la intervención sobre partes enteras de una ciudad no es un hecho nuevo. Lo novedoso es que los grandes proyectos urbanos de última generación son pensados como elementos mediadores entre las exigencias espaciales de las inversiones económicas en las grandes metrópolis y su propia transformación urbana, lo que cuestiona la planificación tradicional por las siguientes razones: realizarse en una temporalidad prologada; ser una obra pública, aunque exista inversión privada; tener orígenes diversos pero estar guiados por una voluntad e idea compartida por los principales actores urbanos (Ezquiaga, 2001).

Este autor hace una clasificación de los grandes proyectos urbanos así:

Los de la primera generación que se caracterizan por tener como objeto la construcción de infraestructuras o servicios urbanos, recuperando y ampliando el concepto de "obra pública"; estar basados en el protagonismo público e intervención directa de gobierno central o municipal; ser concebidos como motores de la transformación urbana y recuperación del medio ambiente; brindar atención prioritaria al espacio público.

Los de la segunda generación se diferencian por diversificar sus los objetos de intervención (conformación de nuevas centralidades, mejoramiento de zonas pobres, etc); romper los límites geográficos de las intervenciones urbanísticas tradicionales llegando incluso a la periferia; introducir criterios de rentabilidad económica incorporando al sector privado en la gestión.

Los de la tercera generación, muchos de ellos grandes proyectos metropolitanos, se singularizan por ser concebidos como motores de desarrollo del espacio metropolitano; promover la recuperación integral de los centros históricos, no solo del patrimo-

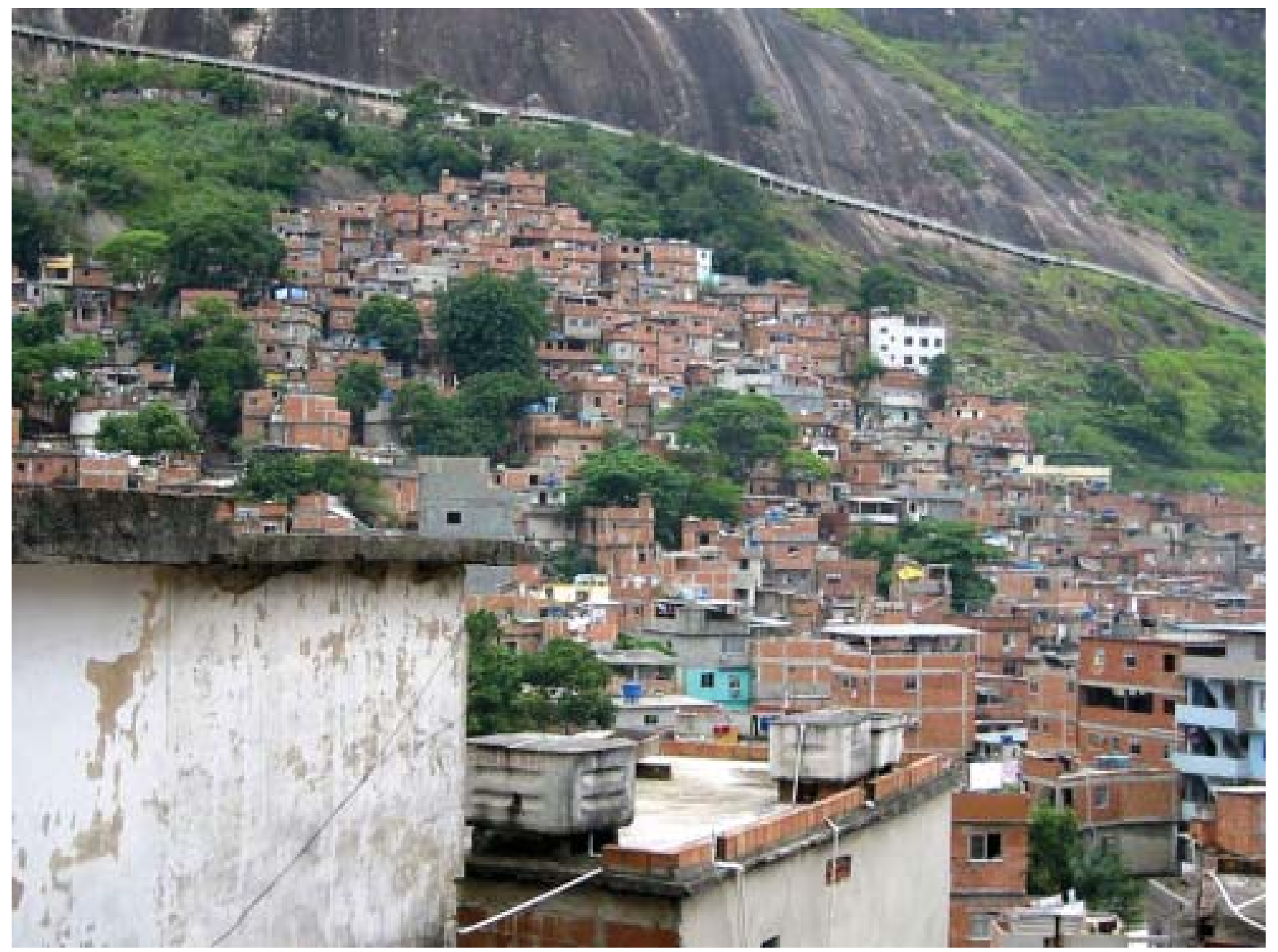

Río de Janei ro: Rehabilitación de grandes áreas de viviendas. 


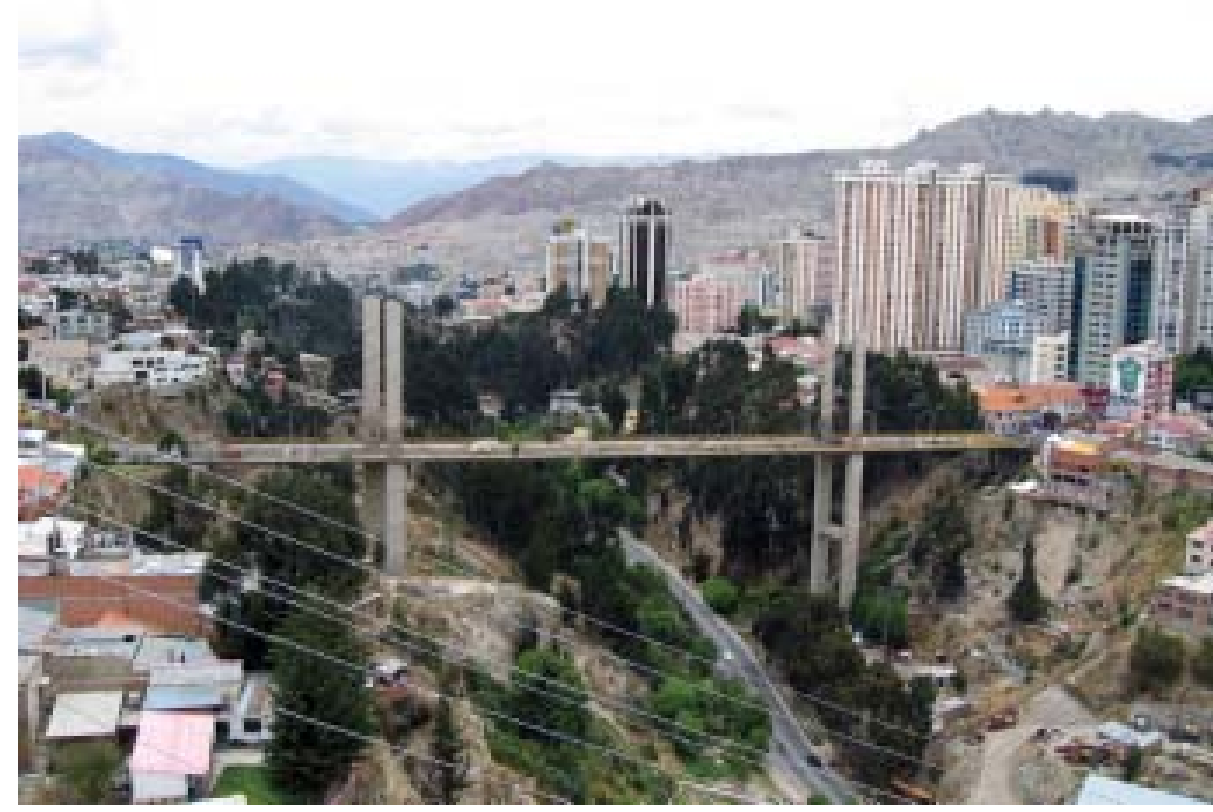

La Paz, Bolivia. La ciudad que se moderniza, salvando enclaustramientos locales.

ciudades latinoamericanas podemos encontrar grandes proyectos urbanos que cumplen con esta característica, entre ellos el Transmilenio construido en Bogotá.

Un gran proyecto urbano o una gran operación urbana no constituyen solo intervenciones únicas en la ciudad de gran dimensión; también pueden estar integrados por un conjunto de pequeñas y medianas intervenciones que siguen una misma lógica, y en algunos casos corresponde a una pieza sola urbana (Garay, 2004). La construcción de más de quinientos pequeños espacios públicos en la ciudad de Nueva York pudiera ser un ejemplo (Kayden, 2000).

Sin embargo muchos grandes proyectos urbanos de ellos han contribuido a una mayor segregación socioespacial en las ciudades y al desplazamiento de población pobre ( procesos de elitización); provocado impactos

nio histórico; contribuir a la transformación la periferia interna creando nuevas centralidades.

La trayectoria de los grandes proyectos urbanos en los Estados Unidos muestra sensibles diferencias con la europea. Las ciudades norteamericanas han sido extraordinariamente independientes de los gobiernos estatales y federal, pero altamente dependientes de las decisiones de inversión federales y del sector privado y con escasa participación ciudadana. Simultáneamente, han hecho énfasis en la competencia entre ellas para captar inversiones privadas. Los instrumentos que tienen las ciudades para conseguir estos objetivos son de dos tipos: incentivos (cambios en la regulación, disminución de impuestos e intereses, etc,); o la construcción de grandes infraestructuras de gran costo, construidas por el sector público, especialmente el Gobierno Federal. Aquí se ubican los llamados megaproyectos en la literatura norteamericana.(Altshuler and Luberoff, 2003).

La cuestión de la participación ciudadana nos conduce a la concepción de grandes proyectos urbanos prevaleciente en Europa. Uno de los ejemplos paradigmáticos de los mismos son las diferentes intervenciones realizadas en la ciudad de Barcelona, especialmente durante los años 90 . Al respecto se ha sostenido (Borja, 1995), que no se deben hacer planteamientos aislados o sectoriales ya que estas intervenciones tienen un carácter polivalente y estratégico. El efecto global no viene dado por la suma de un conjunto de actuaciones sectoriales sino la de una programación y gestión combinadas que comienzan con la participación ciudadana, continua con la polivalencia de las actuaciones y progresa cuando estimula las intervenciones públicas y privadas, pasando del proyecto urbano al proyecto de ciudad. Se insiste en que no debe pensarse que cada proyecto o intervención va a resolver un solo problema, sino que cada uno debe resolver varios problemas, a distintas escalas y con varias funciones (Borja, 2001). En varias ambientales negativos; canalizado el uso de recursos públicos para intervenciones privadas; y sobre todo, creado condiciones para la apropiación individual de la valorización de la tierra urbana, por lo que han sido fuertemente criticados.

Así, los grandes proyectos urbanos han sido ante todo, para varios investigadores, construcciones monumentales, impulsadas en la mayoría de casos por iniciativa gubernamental, destinados a canalizar hacia la empresa privada los beneficios de la recuperación del entorno urbano donde se construyen estos proyectos. Una especie de "show cases" con una gran función mediática para facilitar el consenso de los actores. Estaríamos en el punto límite en que se sostiene que estos proyectos constituyen un instrumento que contribuiría a crear un pensamiento único sobre las ciudades, en que se articulan los intereses económicos con la cultura para competir por el financiamiento internacional (Arantes, 2001), en que las estrategias culturales sirven al crecimiento económico y al control social (Zukin, 1997). Estas críticas podrían acompañar a otras hechas al nivel del urbanismo actual (Ellin, 1996; Smith, 2000).

Incorporados en los planes estratégicos con el objetivo de incrementar la competitividad de las ciudades, tienden a generar procesos de elitización social, valorización de los capitales privados y especulación de la tierra. Es una forma de que el sector público comparta los riesgos de la iniciativa privada, tal como se señalado en una investigación sobre varios proyectos en Europa, que desmistifica los pretendidos efectos multiplicadores en la economía de los grandes proyectos urbanos y que ha concluido que: primero, estos proyectos han servido para obviar las orientaciones y normas de planificación existentes; segundo, los mecanismos de participación son a menudo formales; tercero, los proyectos están en su mayoría pobremente integrados al desarrollo de la ciudad; cuarto, acentúan la polarización socio-económica; y quinto, están asocia- 
dos a modificaciones en la forma de gobernabilidad urbana (Moulaert et al, 2003),

Uno de los mayores desafíos es entonces convertir la concepción de estos grandes proyectos u operaciones en un hecho participativo permanente que vaya construyendo una visión del desarrollo de la ciudad compartido, en una parte integrante de los imaginarios urbanos colectivos construidos por actores múltiples. El hecho de que estén marcados por la incertidumbre y el largo plazo para su ejecución debe, por el contrario, ser aprovechado en sentido positivo y discutir si en lugar de la dicotomía entre partir del proyecto urbano para construir ciudad o a la inversa, no es mejor plantear que se trata de utilizar los dos caminos simultáneamente.

Por otra parte, los grandes proyectos urbanos actuales se deben analizar en el marco del actual proceso de globalización, los cambios en la economía, la sociedad y el Estado, y las contradicciones que genera (Sassen, 1998), considerando las profundas mutaciones que están ocurriendo en las principales ciudades, y las transformaciones en la cultura urbana, en la arquitectura y en el diseño urbano, donde las visiones postmodernistas promueven la construcción de "artefactos urbanos" en oposición a los planes urbanos impulsados por el pensamiento modernista, como se había señalado ya hace algún tiempo (Harvey, 1989).

Los grandes proyectos urbanos en el período actual se desarrollan en el contexto de profundas mutaciones urbanas. La metropolización y la globalización producen un doble efecto: la homogenización y la diferenciación; la primera, que tiende a generar procesos y actores económicos y políticos comunes para todos los países y ciudades; la segunda porque estos actores son más exitosos si son capaces de adaptarse a las circunstancias particulares del lugar y del momento. Por esta última razón la globalización estimula también, paradójicamente, el desarrollo local.

Entre las innumerables reflexiones sobre estas transformaciones urbanas destaquemos una que señala la evolución reciente de las ciudades y de la vida urbana en torno a cuatro rasgos importantes relacionados con los grandes proyectos urbanos (Ascher, 2001): el desarrollo de los medios de transporte y comunicación; la metropolización; la autonomía creciente de los individuos pero dentro de sistemas cada vez más socializados y complejos; y el desarrollo de una sociedad reflexiva marcada por la omnipresencia de la noción de riesgo.

La metropolización y la globalización aparecen así como los ejes centrales de las profundas mutaciones urbanas en curso alrededor de los cuales deben analizarse los grandes proyectos u operaciones urbanas en sus múltiples aspectos, y discutir el papel que se les pretende atribuir como motores del desarrollo de ciudades y regiones al margen del desarrollo de los países, y la dea de que constituyen, por sí mismos, nuevos espacios de participación y construcción de ciudadanía, especialmente en el caso de las ciudades donde predomina la pobreza.

Pero la planificación urbana tradicional no ha desarrollado mecanismos e instrumentos para este fin, ni se ha desarrollado una cultura institucional en el sector público que impulsara la búsqueda de nuevas formas de participación y/o asociación con el sector privado, ni éste último ha modificado su concepción de la tierra como un bien intocable.

$\mathrm{Al}$ respecto de lo último podemos plantear la hipótesis que ha existido, hasta el momento, un doble obstáculo para pensar nuevas formas para la gestión de la tierra en grandes proyectos urbanos:

- La primera, y más importante, es el acentuado sentido de propiedad privada de este bien, interiorizado en casi todos los grupos sociales sin distinción de su nivel de ingresos; - La segunda, que ha comenzado a perder peso en los años recientes, es la alta proporción de la tierra urbana en manos del Estado, en muchos países. En base a esta propiedad se formularon y ejecutaron proyectos urbanos en los que los que el papel del Estado ha sido fundamental en la medida en que poseía el control de la tierra. No obstante, por la debilidad de la intervención del sector público en el desarrollo general de las ciudades y la concepción de la tierra como un bien privado que permite su utilización a discreción por sus propietarios, plasmada en la mayor parte de las legislaciones de los países de América Latina, no es posible encontrar muchos ejemplos innovadores en la gestión de la tierra en proyectos de envergadura llevados a cabo.

En el esquema de planificación tradicional de la ciudad, los grandes proyectos urbanos se ejecutaron siguiendo casi siempre las normas que establecen los marcos regulatorios prevalecientes, sin desarrollarse esquemas de regulación innovadores para este tipo de intervenciones. La mayoría de estos marcos regulatorios se caracterizan por dos rasgos fundamentales: primero, un alto grado de generalidad que no corresponde a la diversidad del desarrollo que caracteriza a toda la ciudad; segundo, una normativa excesivamente detallada y rígida sobre el uso del suelo que no permite adaptarse a las dinámicas de cambio urbanas. Lo anterior explica la continua transgresión de las normativas urbanas vigente en la mayoría de ciudades y es una de las fuentes reconocidas de corrupción. Contribuye, también, a crear muchas dificultades cuando se trata de plantear formas de gestión de la tierra flexibles y adecuadas a objetivos específicos. Los grandes proyectos urbanos se ven enfrentados así a regulaciones de gran generalidad y rigidez que se convierten, muchas veces, en obstáculos insalvables que impiden el cumplimiento de objetivos trazados.

Para solucionar este problema se han ideado varias alternativas: crear regímenes regulatorios especiales, eliminar requisitos vigentes en el marco normativo general, autorizar usos de suelo no permitidos, etc. Estas acciones sin embargo, al adoptarse exclusivamente para estos proyectos, pocas veces se articulan a la regulación vigente para el resto de la ciudad, acentuando las desigualdades urbanas existentes y generando nuevas. Este es uno de los mayores desafíos que enfrenta la gestión de estos proyectos, especialmente si en su ejecución existen diferentes dominios sobre la tierra urbana en que participan distintos actores públicos y privados. La planificación no ha podido, aún, dar una respuesta coherente y sostenible a este problema, especialmente porque opera en un contexto donde se tiende a desregularizar los procesos urbanos y a privatizar la gestión del desarrollo de la ciudad.

Por otra parte, los beneficios buscados con la ejecución de un gran proyecto urbano pueden verse reducidos o anulados por la privatización de servicios urbanos que podría modificar las condiciones de cobertura o elevar los costos; por el abandono del mantenimiento o la construcción de infraestructura antes a cargo de entidades públicas; o por la generación de nuevas instituciones responsables de regulaciones sectoriales sin vinculación entre ellas. También la descentralización, con todos los beneficios que conlle- 
va y sea necesario impulsar, puede dar origen al surgimiento de marcos de regulación territorial fragmentados que pueden afectar los objetivos que se fijan al momento de diseñar una operación urbana de gran envergadura. Hay que recordar que toda descentralización exige el reforzamiento cualitativo de las instituciones centrales.

Es en este contexto que irrumpe la planificación estratégica modificando la situación prevaleciente hacia varias décadas. Este giro no ha tenido, sin embargo, solo connotaciones positivas en la gestión de grandes proyectos urbanos. La razón se explica por las contradictorias consecuencias de algunos de sus planteamientos que han sido llevados a posiciones extremas, entre ellos el rol de este tipo de proyectos en el desarrollo de las ciudades.

Lo que interesa destacar aquí es uno de los puntos críticos de la planificación estratégica en torno a esta problemática: en la medida en que los grandes proyectos urbanos no se articulen a una estrategia para el conjunto de la ciudad, se pueden generar consecuencias negativas de distinta índole, e introducir mayores distorsiones en el siempre contradictorio funcionamiento del mercado de tierra urbana al nivel general.

La planificación estratégica no ha logrado tampoco dar respuesta a los desafíos de crear un nuevo marco regulatorio que apoye el desarrollo urbano integral e incorpore los grandes proyectos urbanos al mismo. Al observarse los casos más exitosos en que se ha utilizado este enfoque es posible identificar dos condiciones que lo han permitido: uno, la existencia de un marco regulatorio moderno, flexible pero con una vocación integral, que trasciende los límites territoriales de la ciudad; dos, la inversión pública en componentes claves de estos proyectos.

El éxito de estos casos, debido a la existencia de condiciones particulares favorables, no ha traducido sin embargo siempre en tendencias de cambio positivas para impulsar una nueva regulación para el conjunto de la ciudad. En esta situación influye, también, el hecho de que los procesos de reforma del Estado han priorizado la privatización sobre la descentralización; las dificultades de ésta debido a su aplicación general sobre una múltiple variedad de condiciones particulares; y el impulso a una desregulación a ultranza sin explorar la posibilidad y necesidad de crear nuevos modelos de regulación para el desarrollo territorial y urbano.

Todo lo anterior, sumado a las exigencias que va imponiendo el proceso de globalización actual, requiere de una renovación de los marcos legales y de los instrumentos normativos de la planificación urbana en general y de los grandes proyectos urbanos en particular. Obviar esta cuestión fundamental solo conduce a fomentar los hábitos de transgresión de la ley y la corrupción tan frecuente en América Latina. Aquí se abre un impor-

\footnotetext{
G randes proyectos de vialidad urbana en d moderno contexto metropolitano, Sao Paulo, Brasil.
}

tante debate que debe incorporar las nuevas regulaciones transnacionales que está produciendo la integración y liberalización creciente de los procesos económicos pero también político-administrativos. Se trata de pasar de la simple sociedad administrativa nacional a sociedades de derecho con fuertes implicaciones transnacionales, donde el enfoque de la negociación tenga un papel central.

Es evidente que los factores de escala y complejidad exigen una mayor, más moderna pero también más decisiva intervención del Estado, lo que contradice a quienes sostienen que el papel de éste último debe limitarse a "facilitar" el desarrollo urbano por parte del mercado. Los grandes proyectos urbanos constituyen así un espacio privilegiado para reflexionar sobre la escala y la complejidad. El crecimiento de la población urbana y su diferenciación social, exigen planes de escala amplia para la construcción de nuevas comunidades y no sólo de edificaciones o conjuntos aislados de gran dimensión. Aquí es útil recordar la antigua crítica a la planificación en gran escala (Douglas, 1973), per explorar los impactos territoriales de las grandes intervenciones en la ciudad (Ferrán, 2001).

Mientras que en el enfoque de la planificación tradicional el papel del sector público es predominante, ocupando un rol rector en la formulación y ejecución de los proyectos urbanos, esta posición tiende a cambiar en la planificación estratégica. En ésta la intervención pública se desplaza de ente ejecutor hacia un papel de facilitador de la gestión de los proyectos. Así, en varios países el predominio del gobierno central ha cedido el puesto al incremento del poder de las colectividades locales y a las organizaciones de ciudadano. La descentralización complejiza más esta situación al introducir nuevos actores y reglas, generando nuevos desafíos: multiplicación de actores e intereses; atomización de los poderes; incertidumbre en la gestión urbana por la larga duración de algunos proyectos; inadaptación de los mecanismos financieros y económicos tradicionales a las nuevas condiciones que produce la desregulación y la privatización. Lo anterior invalida la pretendida función "facilitadora" del Estado.

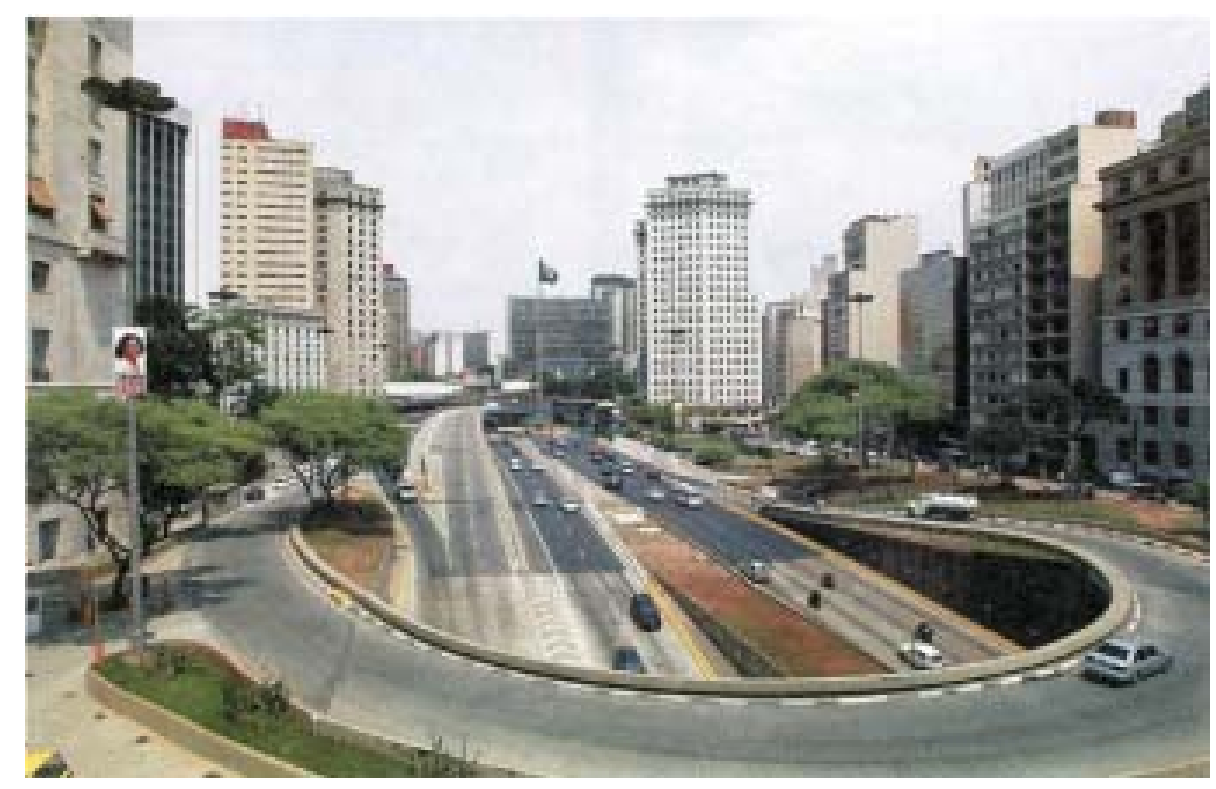


Es esta compleja dinámica el marco de las negociaciones en torno a los grandes proyectos urbanos, donde participan en muchos casos actores multinacionales, nacionales y locales, es un contexto de grandes dificultades pero a la vez de potencialidades para coordinar los intereses y las formas de actuación de un conjunto complejo de actores. Las grandes intervenciones urbanas son por esencia públicas y requieren siempre de la inversión del Estado, lo que marca los límites de acción de las asociaciones público-privadas.

Existen otros puntos que es indispensable discutir alrededor de los grandes proyectos urbanos y la participación social, además de la necesidad de tener una visión integral de la ciudad:

- Primero, para evitar contribuir a la tendencia hacia la fragmentación de la gestión urbana imperante estos días se deben impulsar procesos de establecimiento de consensos y acuerdos en torno al desarrollo de la ciudad en su conjunto y no solo al nivel de proyectos aislados.

- Segundo, este papel de promotor deben hacerlo tanto el gobierno central, el gobierno estatal y los gobiernos locales articuladamente. Debe evitarse un efecto perverso de la descentralización no buscado: la atomización de las responsabilidades públicas.

- Tercero, las organizaciones de la sociedad civil, y particularmente los gremios empresariales, no deben sustituir al sector público en la conducción de la gestión urbana. Hay que evitar la privatización de la misma ya que el mercado no puede cumplir esta función.

- Cuarto, la gestión urbana, además de la conducción pública, requiere de la recuperación de la inversión de este sector, así sea en componentes estratégicos, para poder orientar su desarrollo.

¿Cómo ha planteado el financiamiento de los grandes proyectos urbanos la planificación tradicional y la planificación estratégica?

Para la primera es evidente que el peso mayor de la inversión en este tipo de proyectos, por su escala, recae en el sector público, sea a través del aporte de la tierra, sea a través de este aporte más la inversión en construcción/readecuación de la infraestructura y los equipamientos urbanos. Esta última opción ha prevalecido, por ejemplo, en los Estados Unidos. En América Latina contribuyó en buena medida al endeudamiento externo de muchos países al solicitar préstamos a los organismos financieros multilaterales para realizar grandes intervenciones urbana (Prefeitura do Río de Janeiro, 1999).

Agotada en mucho casos esta posibilidad, o se cesó de promover este tipo de proyectos o se comenzó a buscar la inversión privada, nacional o extranjera. Hay que señalar además que, para muchas ciudades, los montos de las inversiones económicas a invertir en estos proyectos excedía sus capacidades financieras. A lo anterior hay que sumar la debilidad de captación tributaria de los gobiernos locales en muchos países.

Lo anterior fue configurando una creciente incapacidad de gestión de proyectos de gran dimensión en muchas ciudades latinoamericanas, particularmente en las décadas de los años 80 y los años 90 , reduciéndose la función pública al control, cada vez más ineficiente del desarrollo de las ciudades, al mantenimiento sin mayores inversiones ni modernización de los servicios públicos, y a la ejecución de proyectos puntuales.
Surgen así la asociaciones público-privadas para impulsar grandes proyectos urbanos. Existe una amplia gama de asociaciones público-privadas. Existe una gama de situaciones intermedias en las que los sectores público y privado se involucran más profundamente en la sociedad y contribuyen con financiamiento, capacidad de gestión y de coordinación con otras entidades públicas. En este tipo de sociedades, los aportes de recursos privados son de riesgo, ya que no están garantizados ni asegurados por el sector público. Entre esas sociedades figuran empresas de capital mixto, fideicomisos y corporaciones para el desarrollo urbano (Rojas, 2004).

Este modelo de asociación responde bien a las necesidades de la recuperación de áreas urbanas centrales dado que combinan eficazmente capacidades tanto del sector público como del sector privado para emprender tareas tan variadas como: consolidar suelo por medio de compras e intercambios de parcelas; captar para fines públicos los aumentos del valor del suelo resultantes de las acciones de recuperación; construir o rehabilitar espacio para usos residenciales, de servicios, comerciales y productivos, y luego comercializarlo y administrarlo; y asegurar la participación de la comunidad en las decisiones y beneficios de los procesos de recuperación (Sandroni, 2004).

Pero el caso de la corporación para el desarrollo de Bilbao Metropolitano, RIA 2000, es un ejemplo de resultados contradictorios a pesar de su innegable éxito. Creada como una firma privada por iniciativa del gobierno central, opera en la práctica como una agencia cuasi pública, cuyo objetivo es manejar una serie de operaciones de renovación urbana en el Area Metropolitana de Bilbao, contando con un considerable poder para manejar tierras y fondos públicos, que se arriesgan para impulsar proyectos que propician una mayor valorización de las tierras y el capital privado. No obstante su potencial como agencia ejecutiva y de coordinación entre diferentes actores, su estatus como empresa privada ha generado crítica por papel en la privatización de la planificación urbana, la falta de responsabilidad política, su énfasis en el desarrollo de sitios de elevado potencial comercial y alto retorno financiero, así como en la priorización de los aspectos físicos de la renovación urbana (Rodríguez, 1995 y 2002).

\section{Tendencias actuales hacia la privatización de la gestión urbana}

Queremos concluir este trabajo partiendo de esta afirmación: la concepción acrítica que mira a los grandes proyectos urbanos conducidos por el sector privado a través de asociaciones público-privadas, como el elemento motor del desarrollo de las ciudades, es una de las expresiones más importantes de la visión neoliberal de la gestión urbana, en la cual el papel de la institucionalidad pública y el interés general tienden a desvanecerse en función de los intereses privados bajo el manto de la necesidad de incrementar la competitividad de las ciudades exigida por la globalización. Lo anterior no invalida la necesidad de pensar, dada la creciente complejidad de los fenómenos urbanos, en impulsar intervenciones urbanas de gran dimensión de distinto tipo, pero que efectivamente generen ciudades más justas y equitativas. Este es uno de los desafíos centrales de la gestión urbana en el momento actual. 
Pero los impulsos hacia la privatización de la gestión urbana van más allá de las grandes intervenciones. Estas se combinan con cambios en los procesos de planificación, regulación, administración e inversión en las ciudades. Examinemos lo anterior.

Respecto a la planificación, su continuo debilitamiento que responde a las políticas neoliberales es general al nivel de la toda la ciudad, sigue siendo sustituida por la sumatoria de acciones atomizadas en todas las escalas, desde los grandes hasta los pequeños proyectos. La sustitución de los antiguos marcos regulatorios urbanos a través de normativas específicas, especialmente las dedicadas para los grandes proyectos, justificada por la obsolescencia y carácter excesivamente general y normativo de los primeros, está conduciendo a la fragmentación de esta función pública clave del desarrollo de las ciudades, tendencia que se acentúa por el desmantelamiento de los aparatos estatales, lo que, a su vez, contribuye a debilitar la administración pública urbana. Respecto a la inversión, la reducción drástica de las inversiones públicas en las ciudades deja cada vez más espacio al predominio de las inversiones privadas.

Este es el panorama que predomina en el ámbito urbano de la mayoría de países latinoamericanos, reforzando la tendencia a la privatización de gestión urbana. Podría plantearse, y existen casos exitosos, de una adecuada combinación de inversiones públicas y privadas. Sostenemos, sin embargo, que éstas tienen como requisitos indispensables los siguientes:

- primero, aunque deba modernizarse y flexibilizarse, la planificación urbana debe mantener un papel importante en el desarrollo de las ciudades;

- segundo, debe evitarse la regulación urbanística caso por caso y la discresionalidad en la aplicación de las normas; aunque están deban ser repensadas radicalmente, hay que mantener su carácter genérico;

- tercero, la administración del desarrollo de las ciudades debe ser una función exclusivamente pública, lo que no excluye la consulta de las decisiones con el sector privado;

- cuarto, hay que recuperar la importancia de las inversiones públicas en el desarrollo urbano; aun cuando las restricciones económicas imponen serias limitaciones, estas deben mantenerse en los sectores y servicios estratégicos.

Se trata, entonces, de transformar y modernizar las formas tradicionales de gestión urbana pero evitando su privatización. El Estado no debe ser simplemente un facilitador de las intervenciones e inversiones privadas, aunque deba cumplir esta función. Debe conducir, dirigir el desarrollo de las ciudades para garantizar niveles mínimos de equidad y sostenibilidad. En este proceso de conducción un aspecto fundamental es la contribución a la construcción de un proyecto de ciudad, otro requisito indispensable para que las grandes intervenciones urbanas adquieran sentido.

Hay una última cuestión que es importante discutir: las escalas de la gestión urbana. En momentos en que le lucha por la descentralización y el desarrollo local, hay que evitar la atomización de la gestión urbana, lo que contribuye, así sea indirecta e involuntariamente a su privatización. Muchos procesos urbanos, especialmente algunos de los principales como por el ejemplo el transporte, responden a escalas que no es posible segmentar, por lo que la atomización de su gestión a escalas inferiores torna su gestión ineficiente, dando fuerza a quienes propugnan por la privatización de esta función. Evidentemente hay procesos urbanos loca- les y la clave está en identificarlos y diferenciarlos de los procesos mayores.

Los grandes proyectos urbanos, precisamente por sus características principales: su complejidad y su escala, pueden contribuir tanto a la privatización como a la recuperación de la esencia pública de la gestión urbana. Pensarlos aisladamente refuerza la primera y negativa tendencia.

\section{BIBLIOGRAFIA}

Ascher, François (2001): "la nouvelle révolution urbaine: de la planification au management stratégique urbain”, en Fabriquer la ville. Outils et méthodes:les aménageurs proposent, Masboungi, Ariella (coordinatrice), La Documentation FranVaise, Paris.

Banco Mundial (2000): Cities in Transition, Washington.

Borja, Jordi (1995): Barcelona. Un modelo de transformación urbana, Programa de Gestión Urbana, Quito.

Ellin, Nain (1996): Postmodern Urbanism, Basil Blackwell, London.

Ezquiaga, José María (2001): "Projetos de transformaçao urbana na Madri do fim do século", Cidades em transformaçao: entre o plano e o mercado, P. Abramo (organizador), IPPURUniversidade Federale do Rio de Janeiro, Rio de Janeiro.

Garay, Alfredo (2004): "El montaje de grandes intervenciones de urbanismo", en Grandes proyectos urbanos, Mario Lungo (compilador), UCA Editores / Lincoln Instittute of Land Policy, San Salvador.

Harvey, David (1996): "Do gerenciamento ao empresariamento: a transformaçao da administraçao urbana no capitalismo tardio", en Espaço e Debates \# 39, NERU, Sao Paulo.

Harvey, David (1989): The Condition of Postmodernity, Basil Blackwell, Oxford.

Kayden, Jerold (2000): Privately Owned Public Space. The New York City Experience, John Wiley and sons, New York.

Lee, Douglas (1973): "Requiem for large-scale planning models", Journal of the American Institute of Planners \# 73.

Lungo, Mario (1992): Procesos urbanos, Itsmo Editores, San Salvador.

Lungo, Mario (2004): Grandes proyectos urbanos, Mario Lungo (compilador), UCA Editores / Lincoln Instittute of Land Policy, San Salvador.

Prefeitura de Sqao Paulo (1991): "Plano Diretor do Municipio de Sao Paulo" (Projeto de Lei), Diário Oficial do Municipio de Sao Paulo, $\mathrm{N}^{\circ} 37$, Sao Paulo.

- Prefeitura da Cidade do Rio de Janeiro (1999): Cidade Inteira. A política Habitacional da Cidade do Rio de Janeiro, Rio de Janeiro.

Rodríguez, Arantxa (2002): "Reinventar la ciudad: milagros y espejismos de la revitalización urbana en Bilbao", en Lan Harremanak \# 6, Universidad del país Vasco, Bilbao..

Rojas, Eduardo (2004): "La recuperación de áreas centrales, una oportunidad de desarrollo urbano sostenible", en Grandes proyectos urbanos, Mario Lungo (compilador), UCA Editores / Lincoln Institute of Land Policy, San Salvador.

Sandroni, Paulo (2004): "Formas de financiamiento de grandes proyectos urbanos", en Grandes proyectos urbanos, Mario Lungo (compilador), UCA Editores / Lincoln Institute of Land Policy, San Salvador.

Smith, Neil (2000): “Transformando la escala de las ciudades: globalización y urbanismo neoliberal”, en Globalización: transformaciones urbanas, precarización social y discriminaciones de género, L.M. García, F. Sabaté, M.A. Mejías y V. Martín (editores), Departamento de Geografía, Universidad La Laguna, Tenerife. 